Supporting Information

\title{
A Bioinspired Synthesis of Sedaxane Metabolite Using Catalytic Vanadyl Acetylacetonate and Molecular Oxygen
}

Sameer Tyagi, ${ }^{*}{ }^{\dagger}$ Christopher D. Cook, ${ }^{\dagger}$ Dana A. DiDonato, ${ }^{\dagger}$ Jeffery A. Key, ${ }^{\dagger}$ Bruce P. McKillican, ${ }^{\dagger}$ William J. Eberle, ${ }^{\dagger}$ Timothy J. Carlin, ${ }^{\dagger}$ David A. Hunt, ${ }^{\dagger}$ Samantha J. Marshall, ${ }^{\ddagger}$ Nichola L. Bow ${ }^{\ddagger}$

${ }^{\dagger}$ Product Metabolism Analytical Sciences, Syngenta Crop Protection, LLC, 410 Swing Road, Greensboro, NC 27409, U.S.A. ${ }^{\ddagger}$ Product Metabolism Analytical Sciences, Syngenta Crop Protection, Jealott's Hill International Research Center, Bracknell, Berkshire, RG 42 6EY, United Kingdom Supplementary Information - Table of Contents

${ }^{1} \mathrm{H}$ NMR Spectra S2

${ }^{13} \mathrm{C}$ NMR Spectra S11 


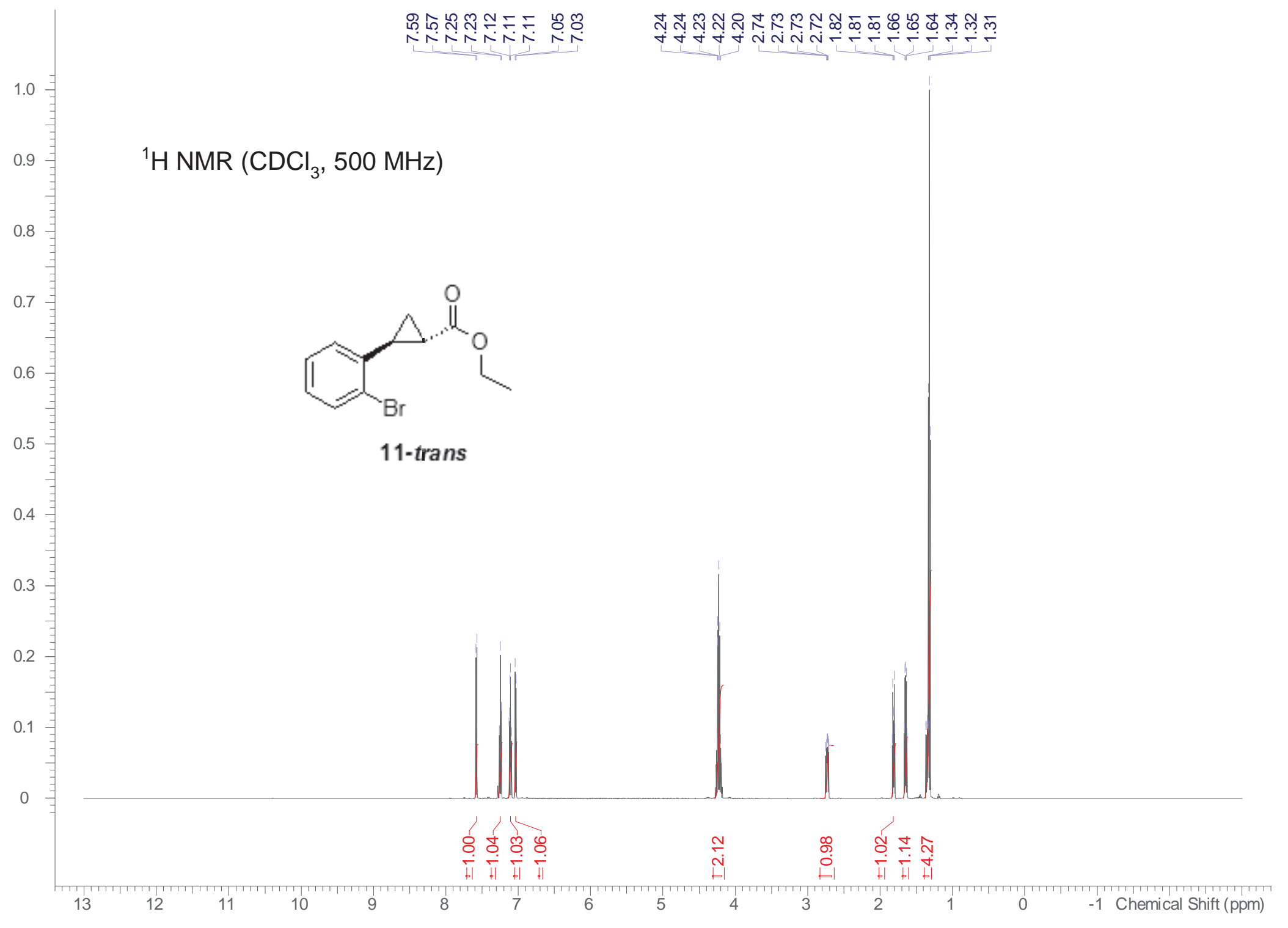




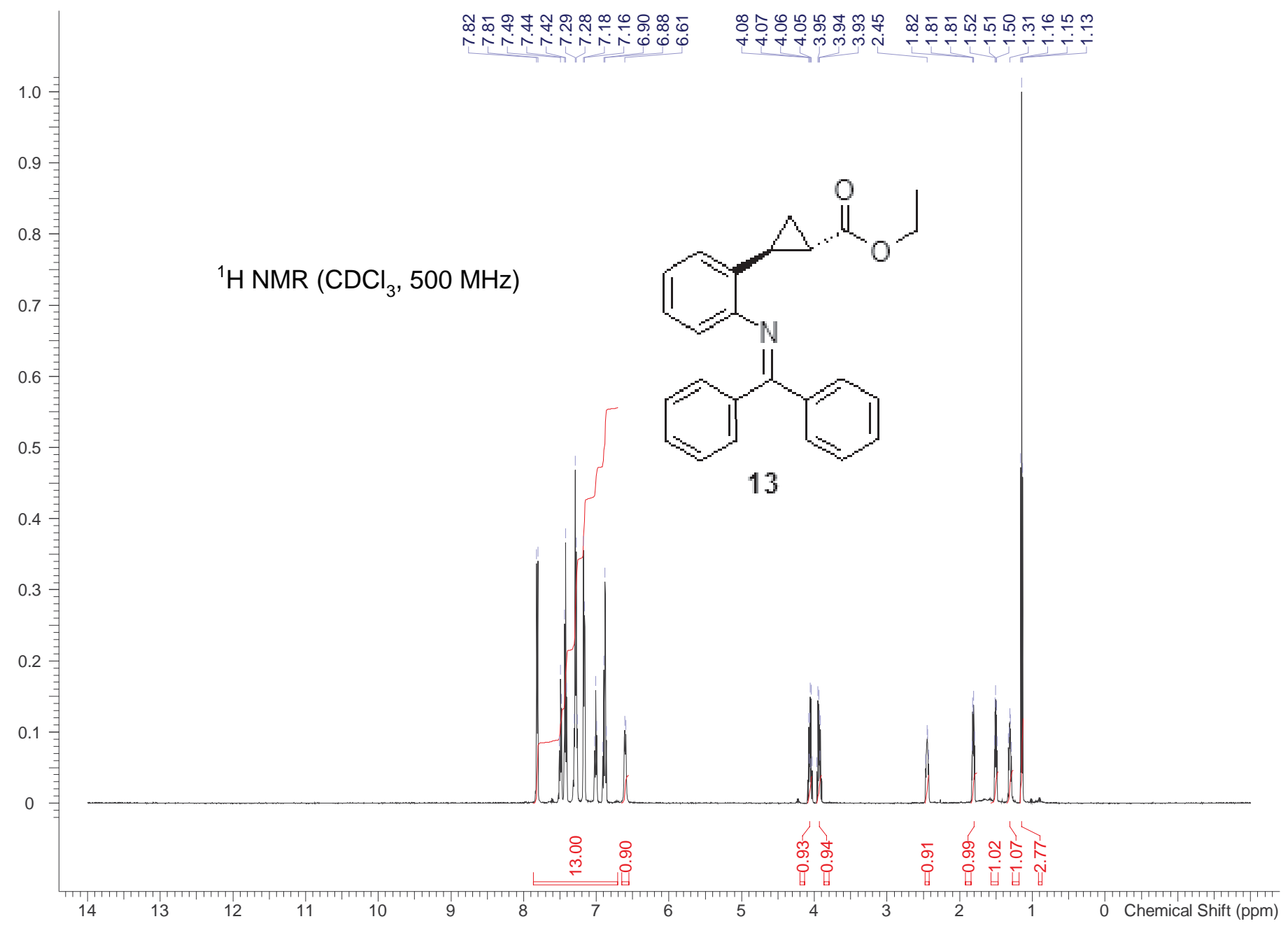




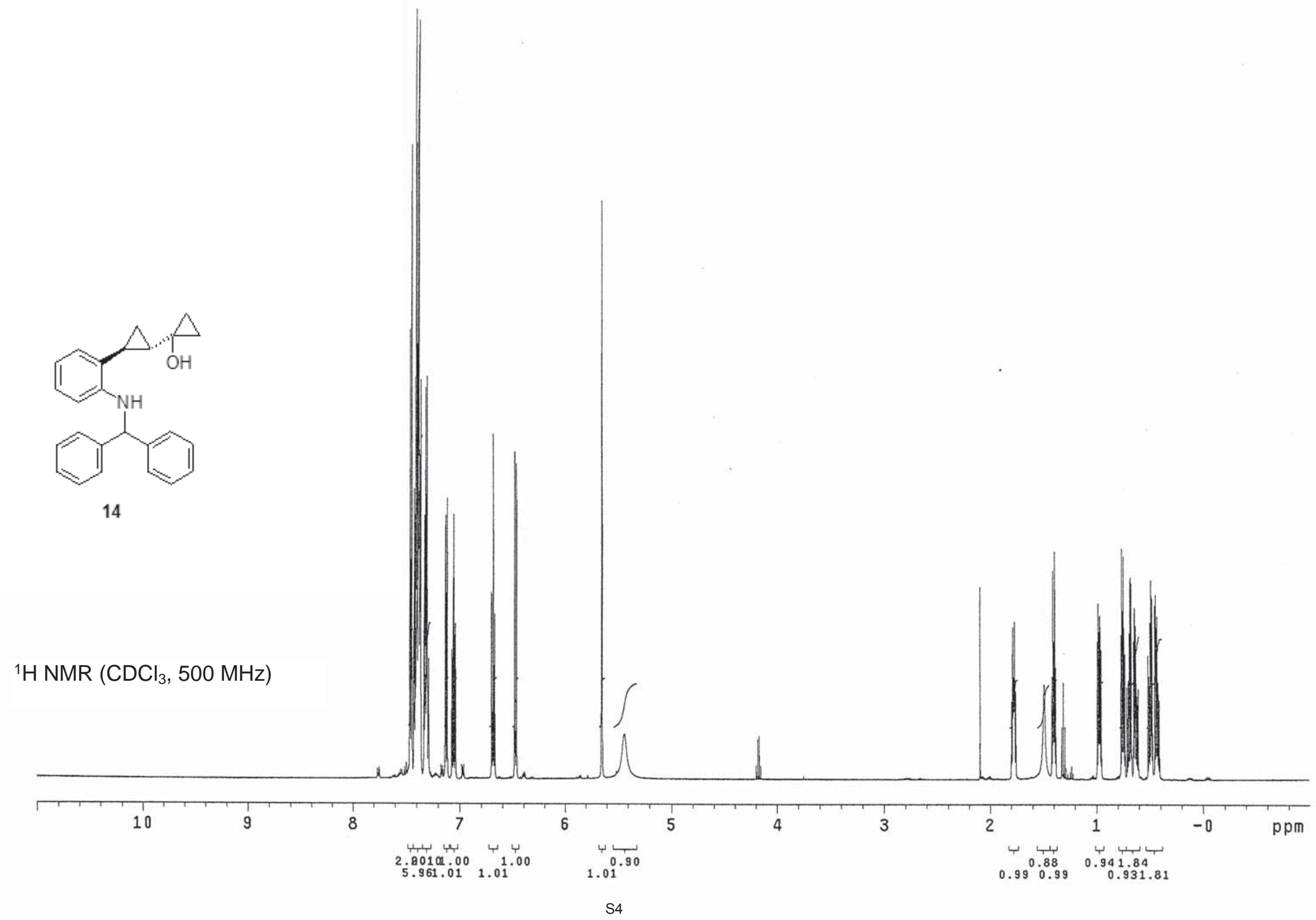


FREQUENCY PPM $3725.138 \quad 7.458$ $\begin{array}{lll}3717.821 \quad 7.443 & \end{array}$ $3707.578 \quad 7.423$ $3705.748 \quad 7.419$ $3703.919 \quad 7.415$ (3697.700 7.403 $3687.091 \quad 7.382$ $3684.164 \quad 7.376$ $3683.067 \quad 7.374$ $3681.237 \quad 7.370$ $3679.408 \quad 7.366$ $3677.945 \quad 7.363$ $3676.481 \quad 7.361$ $\begin{array}{lll}3674.652 & 7.357\end{array}$ 361.8587 .357 3060.3857 .331 $3660.385 \quad 7.328$ 365.5567 .325 $364.897 \quad 7.317$ $3653.434 \quad 7.314$ $3650.873 \quad 7.309$ $3649.775 \quad 7.307$ $\begin{array}{lll}3647.946 & 7.303\end{array}$ $3641.727 \quad 7.291$ $\begin{array}{lll}3640.630 & 7.289\end{array}$ $3639.166 \quad 7.286$ $3638.069 \quad 7.284$ $\begin{array}{lll}3561.243 \quad 7.130 & \end{array}$ $3553.926 \quad 7.115$ $3531.976 \quad 7.071$ $\begin{array}{lll}3530.879 & 7.069\end{array}$ $\begin{array}{lll}3524.660 & 7.057\end{array}$ 3523.9287 .055 $3523.196 \quad 7.054$ $3516.611 \quad 7.040$ 3515.5147 .040 $\begin{array}{ll}3348.327 & 6.704\end{array}$

$\begin{array}{rc}\text { HEIGHT } & \text { INDEX } \\ 88.5 & 40 \\ 124.5 & 41 \\ 38.6 & 42 \\ 57.0 & 43 \\ 19.2 & 44 \\ 151.2 & 45 \\ 129.0 & 46 \\ 124.8 & 47 \\ 85.7 & 48 \\ 144.3 & 49 \\ 149.1 & 50 \\ 64.4 & 51 \\ 34.4 & 52 \\ 61.3 & 53 \\ 42.2 & 54 \\ 78.4 & 55 \\ 11.7 & 56 \\ 28.7 & 57 \\ 51.7 & 58 \\ 26.2 & 59 \\ 64.7 & 60 \\ 76.9 & 61 \\ 20.5 & 62 \\ 24.5 & 63 \\ 79.1 & 64 \\ 16.7 & 65 \\ 23.4 & 66 \\ 13.9 & 67 \\ 12.5 & 68 \\ 51.7 & 69 \\ 55.2 & 70 \\ 25.2 & 71 \\ 23.4 & 72 \\ 51.9 & 73 \\ 48.3 & 74 \\ 46.4 & 75 \\ 30.2 & 76 \\ 27.2 & 77 \\ 36.2 & 78\end{array}$

$\begin{array}{rlrc}\text { FREQUENCY } & \text { PPM } & \text { HEIGHT } & \text { INDEX } \\ 3347.595 & 6.702 & 35.9 & 79 \\ 3341.010 & 6.689 & 67.7 & 80 \\ 3339.912 & 6.687 & 67.2 & 81 \\ 3333.693 & 6.674 & 31.9 & 82 \\ 3332.596 & 6.672 & 31.6 & 83 \\ 3238.210 & 6.483 & 64.2 & 84 \\ 3230.893 & 6.468 & 61.9 & 85 \\ 2821.157 & 5.648 & 113.8 & 86 \\ 2715.065 & 5.436 & 8.7 & 87 \\ 2089.119 & 4.183 & 7.7 & 88 \\ 2081.803 & 4.168 & 8.4 & 89 \\ 1046.487 & 2.095 & 37.6 & 90 \\ 897.592 & 1.797 & 15.1 & 91 \\ 892.104 & 1.786 & 29.6 & 92 \\ 888.811 & 1.779 & 19.4 & 93 \\ 886.982 & 1.776 & 20.0 & 94 \\ 883.690 & 1.769 & 30.6 & 95 \\ 878.202 & 1.758 & 17.1 & 96 \\ 746.867 & 1.495 & 18.5 & 97 \\ 709.552 & 1.421 & 20.2 & 98 \\ 704.430 & 1.410 & 40.9 & 99 \\ 700.772 & 1.403 & 26.8 & 100 \\ 699.308 & 1.400 & 25.3 & 101 \\ 695.650 & 1.393 & 44.6 & 102 \\ 690.528 & 1.382 & 21.9 & 103 \\ 662.725 & 1.327 & 10.1 & 104 \\ 655.774 & 1.313 & 18.9 & 105 \\ 648.457 & 1.298 & 10.2 & 106 \\ 496.270 & 0.994 & 26.8 & 107 \\ 491.514 & 0.984 & 34.2 & 108 \\ 490.416 & 0.982 & 31.8 & 109 \\ 487.489 & 0.976 & 28.7 & 110 \\ 485.660 & 0.972 & 31.6 & 111 \\ 482.734 & 0.966 & 31.8 & \\ 482.002 & 0.965 & 29.7 & \\ 476.880 & 0.955 & 25.3 & \\ 384.324 & 0.769 & 28.1 & \\ 379.202 & 0.759 & 45.2 & \\ 375.544 & 0.752 & 32.7 & \\ & & & \end{array}$
HEIGHT

$\begin{array}{ll}370.422 & 0.742\end{array}$

.78
.704

$\begin{array}{lll}349.935 & 0.701 & 19.4\end{array}$

$\begin{array}{lll}344.813 & 0.690 & 28.5\end{array}$

$\begin{array}{lll}340.789 & 0.682 & 38.9\end{array}$

$\begin{array}{lll}339.326 & 0.679 & 39.6\end{array}$

$\begin{array}{lll}334.936 & 0.671 & 38.4 \\ 324.327 & 0.649 & 26.9\end{array}$

$319.205 \quad 0.639$

$\begin{array}{lll}314.449 & 0.630 & 29.8\end{array}$

$\begin{array}{lll}312.986 & 0.627 & 18.7\end{array}$

$\begin{array}{lll}308.962 & 0.619 & 14.7\end{array}$

$303.230 \quad 0.617 \quad 16.1$

$\begin{array}{lll}258.110 & 0.517 & 24.2\end{array}$

$\begin{array}{lll}253.720 & 0.508 & 20.1\end{array}$

$\begin{array}{lll}251.891 & 0.504 & 18.1\end{array}$

$\begin{array}{llll}247.867 & 0.496 & 30.5\end{array}$

$\begin{array}{lll}246.769 & 0.494 & 17.1\end{array}$

$\begin{array}{lll}243.111 & 0.487 & 39.0 \\ 241.648 & 0.484 & 35.6\end{array}$

$\begin{array}{lll}241.648 & 0.484 & 35.6\end{array}$

$225.551 \quad 0.452 \quad 33.8$

$\begin{array}{lll}221.161 & 0.443 & 35.2\end{array}$

$\begin{array}{lll}220.063 & 0.441 & 36.0 \\ 216.405 & 0.433 & 18.7\end{array}$

$\begin{array}{llll}214.942 & 0.430 & 31.6\end{array}$

$\begin{array}{lll}210.917 & 0.422 & 19.0\end{array}$

$209.820 \quad 0.420 \quad 18.3$

15.2 


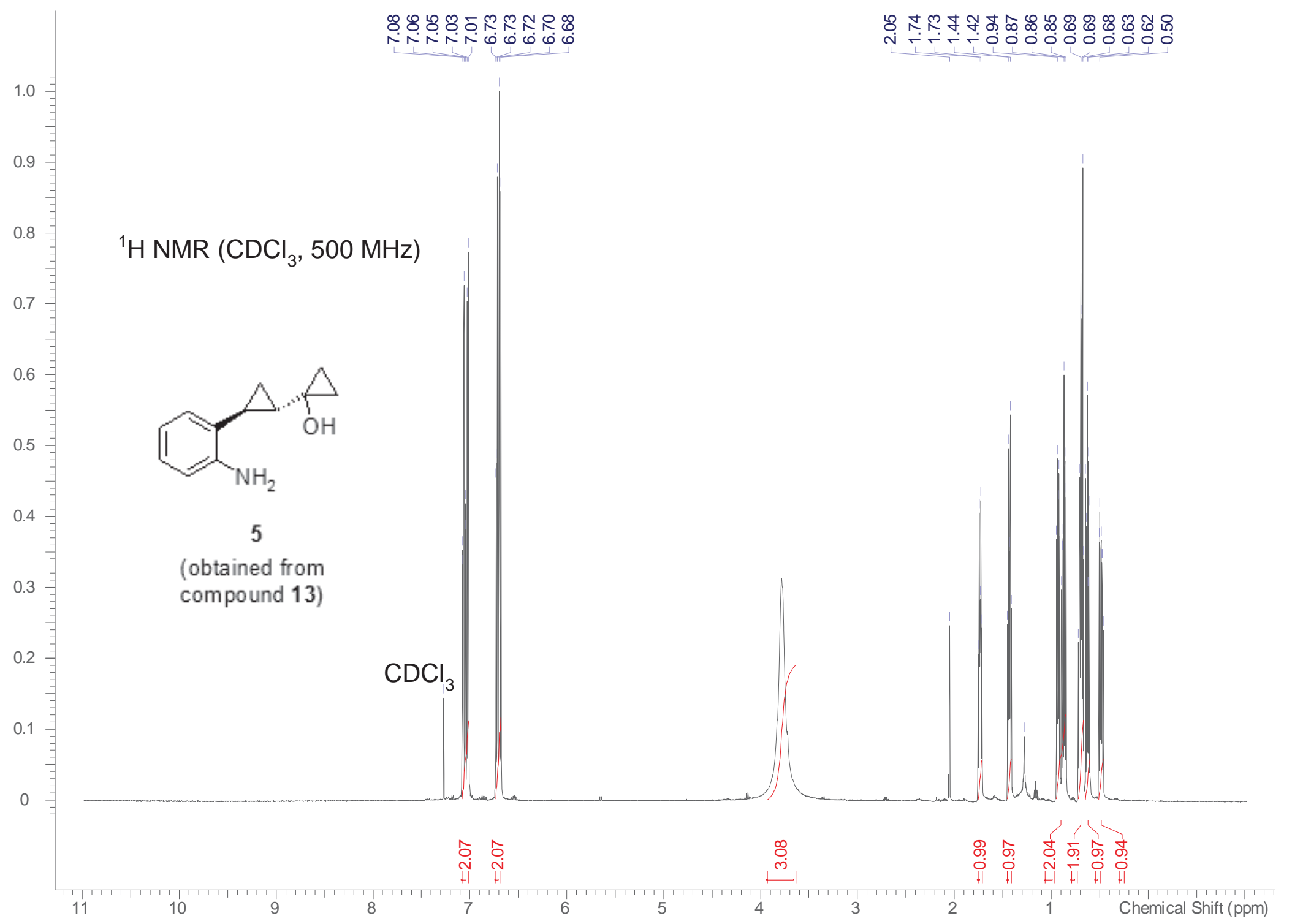




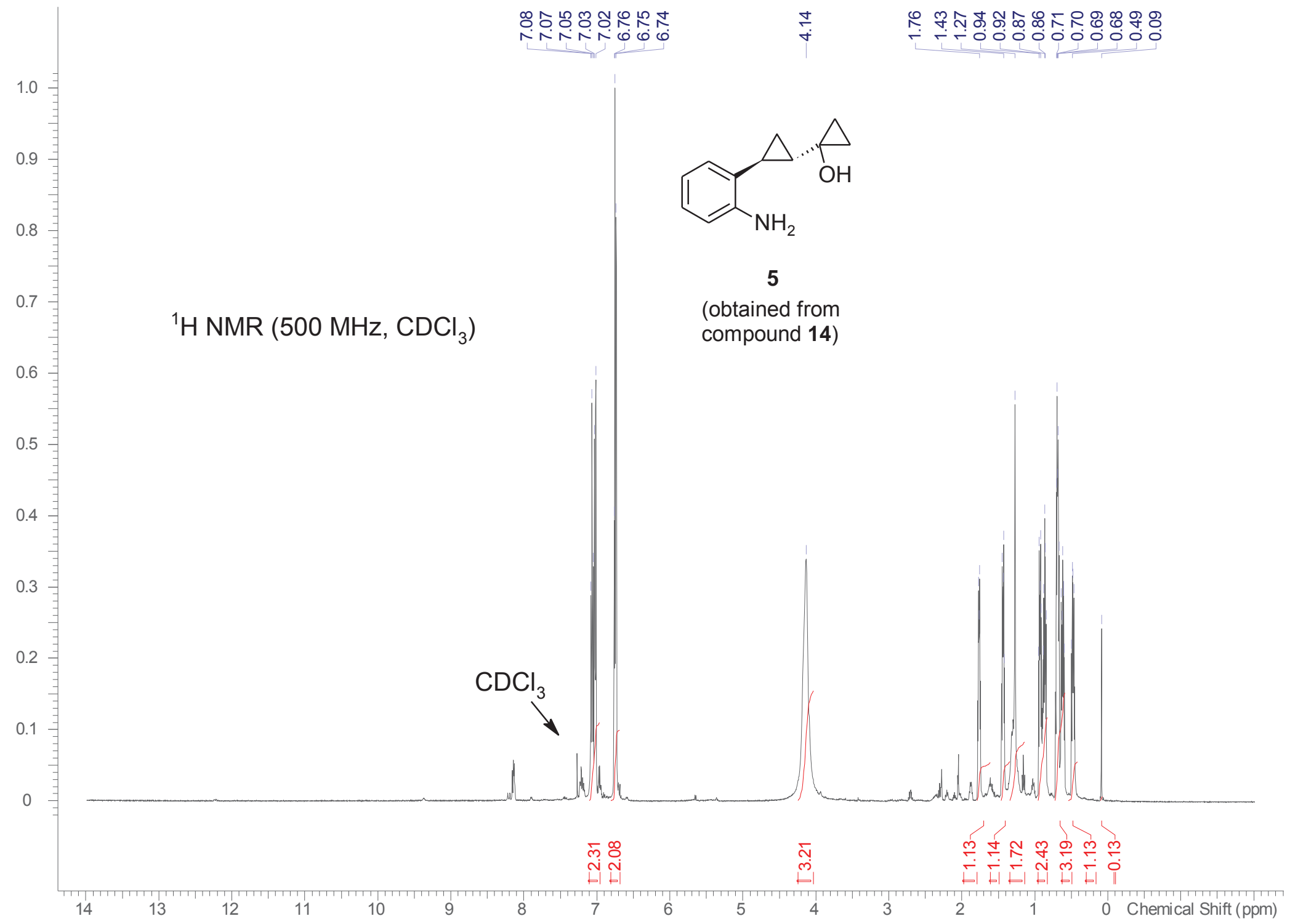




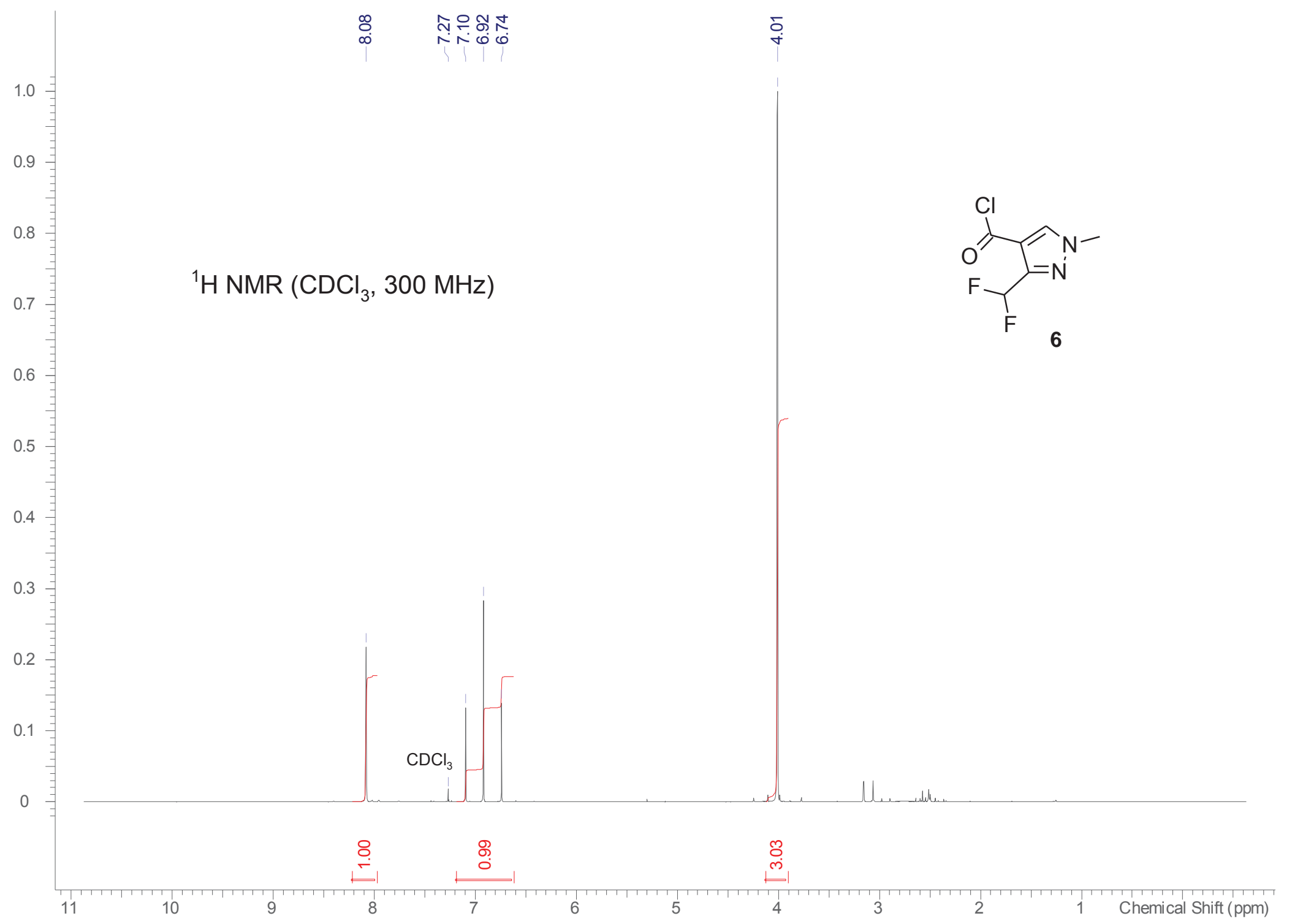




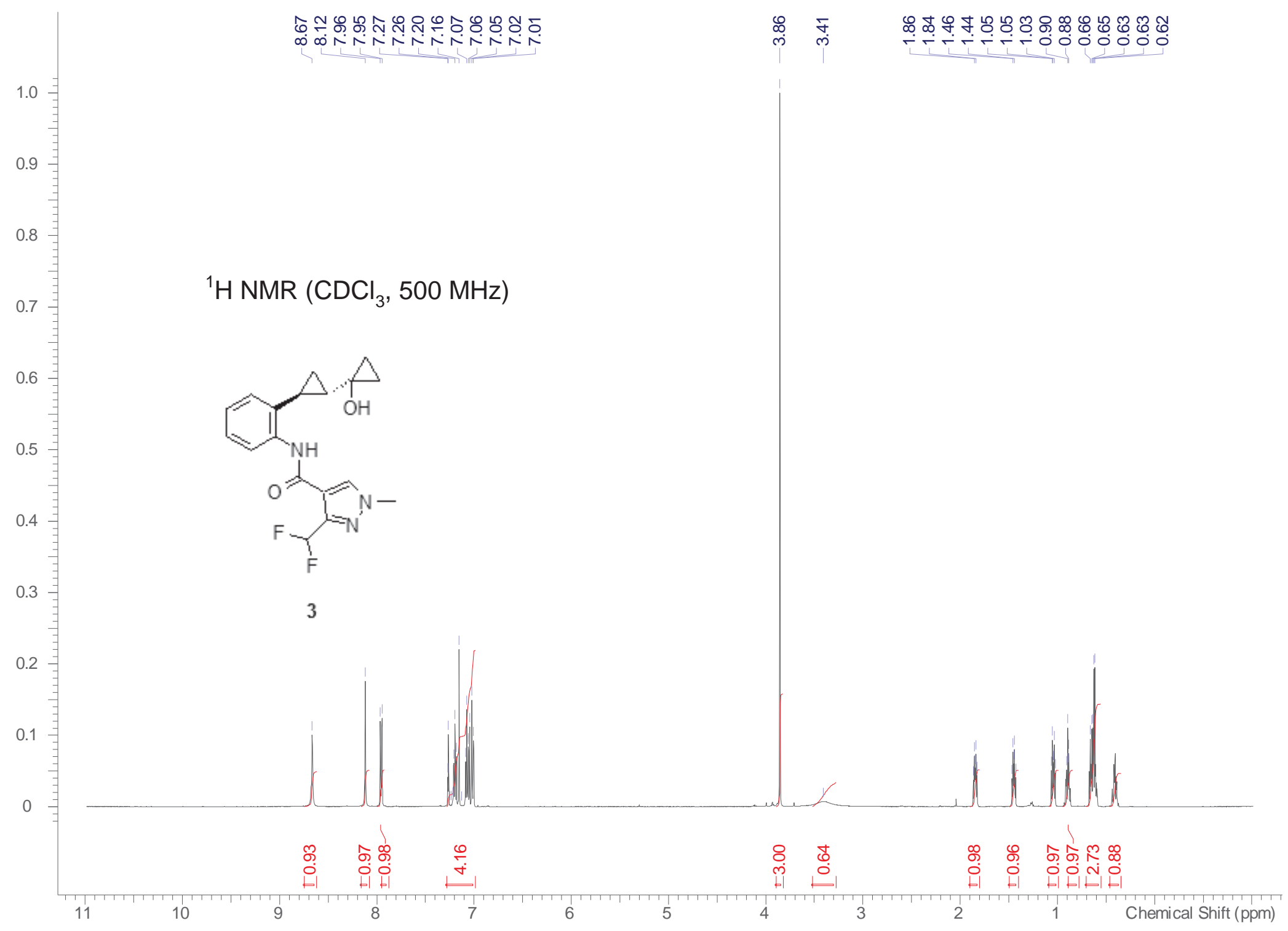




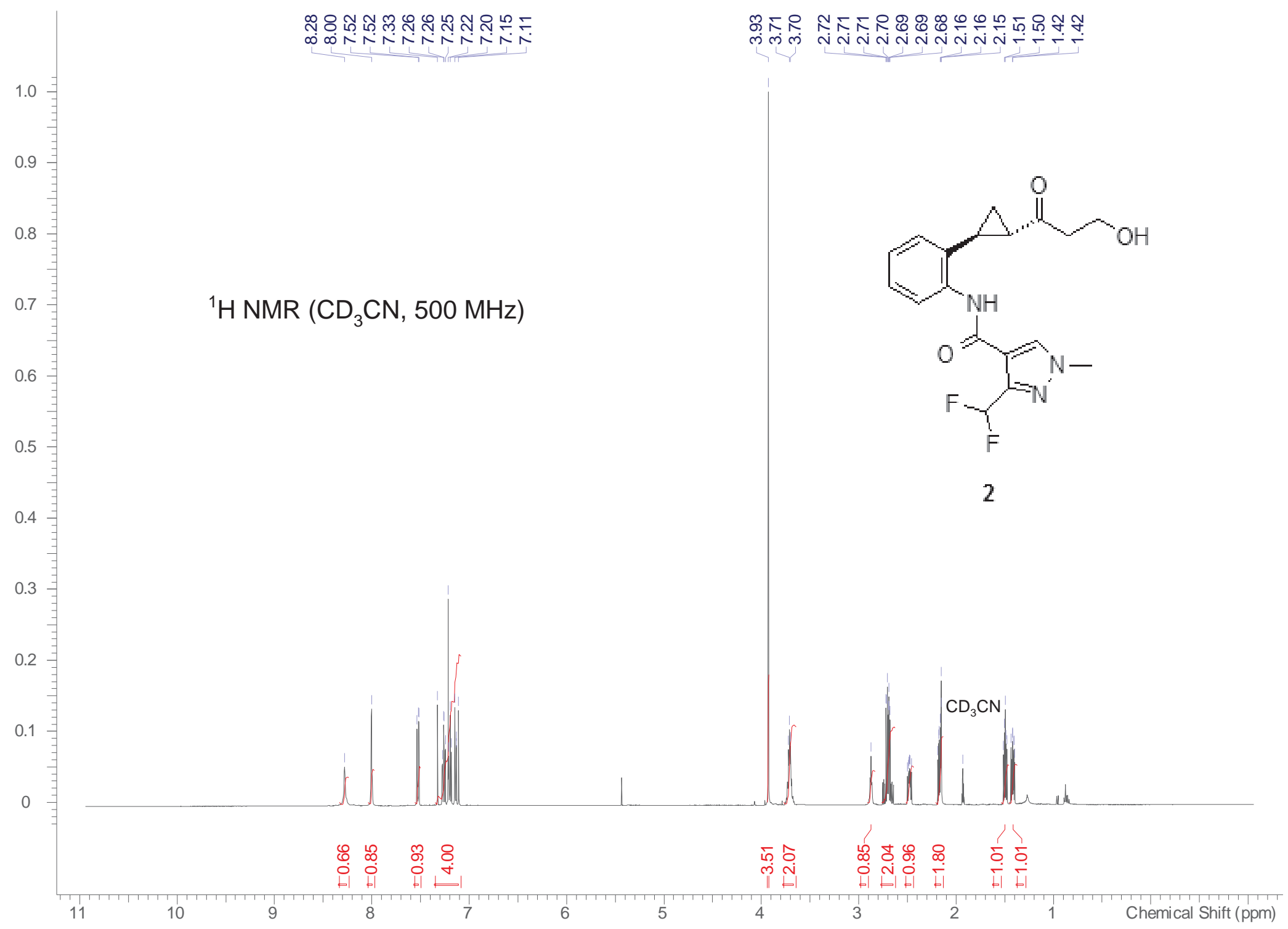




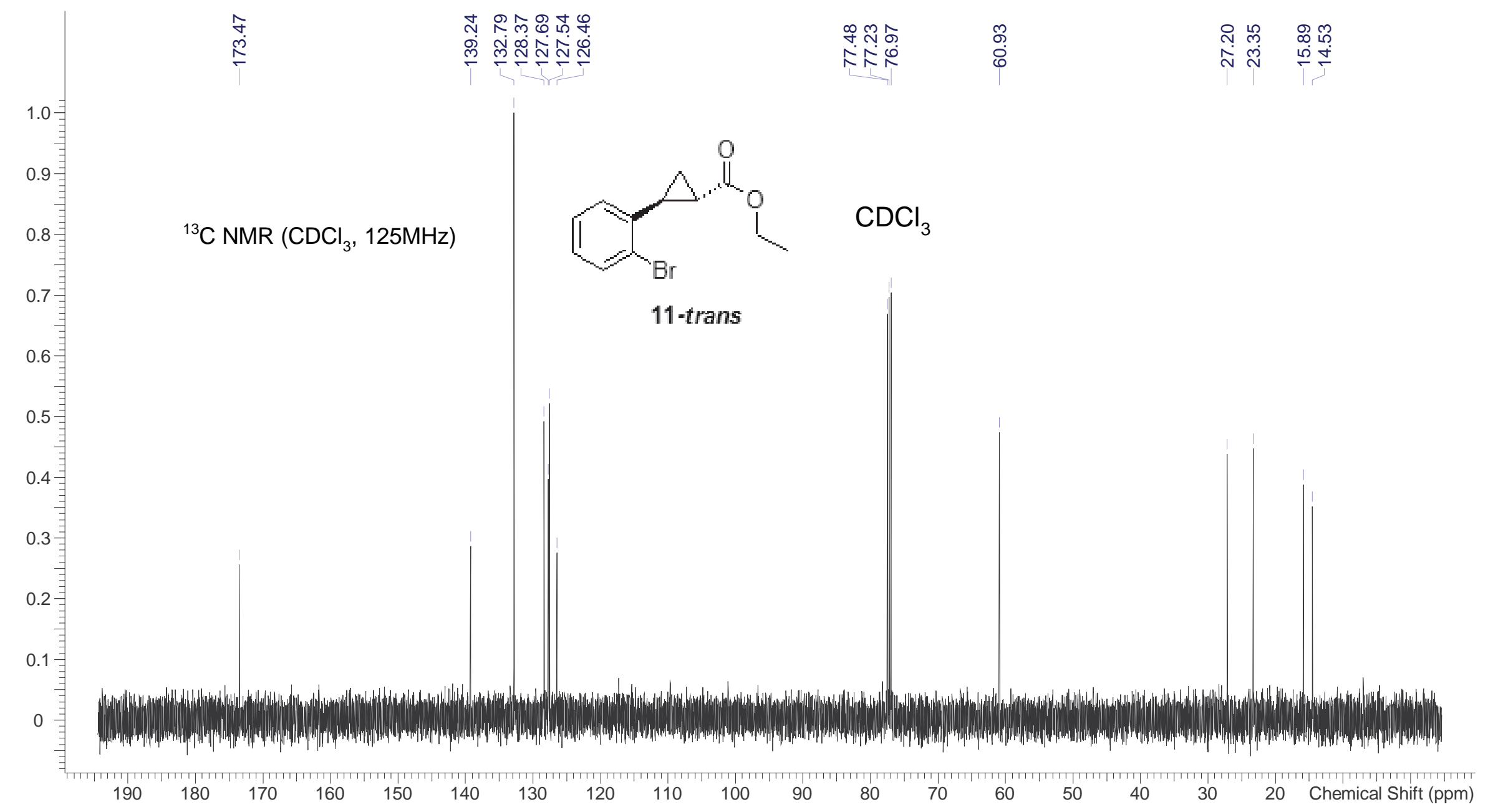




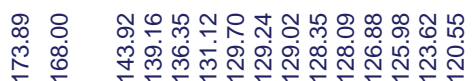

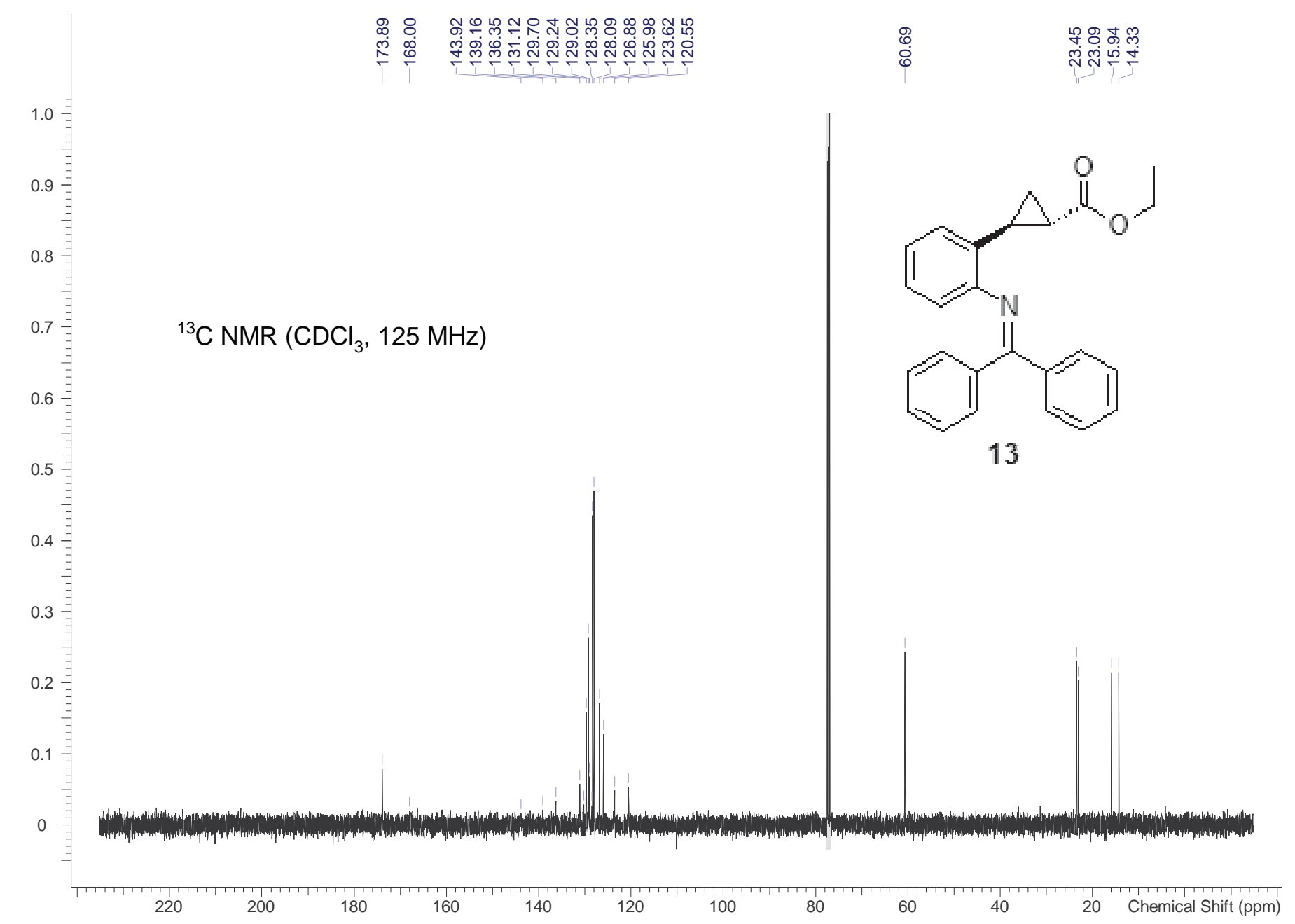




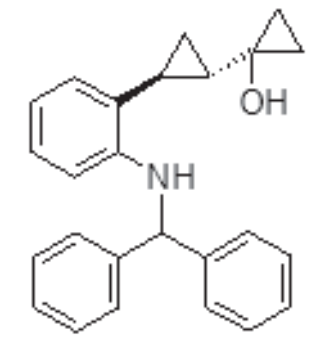

14
\%马ำ
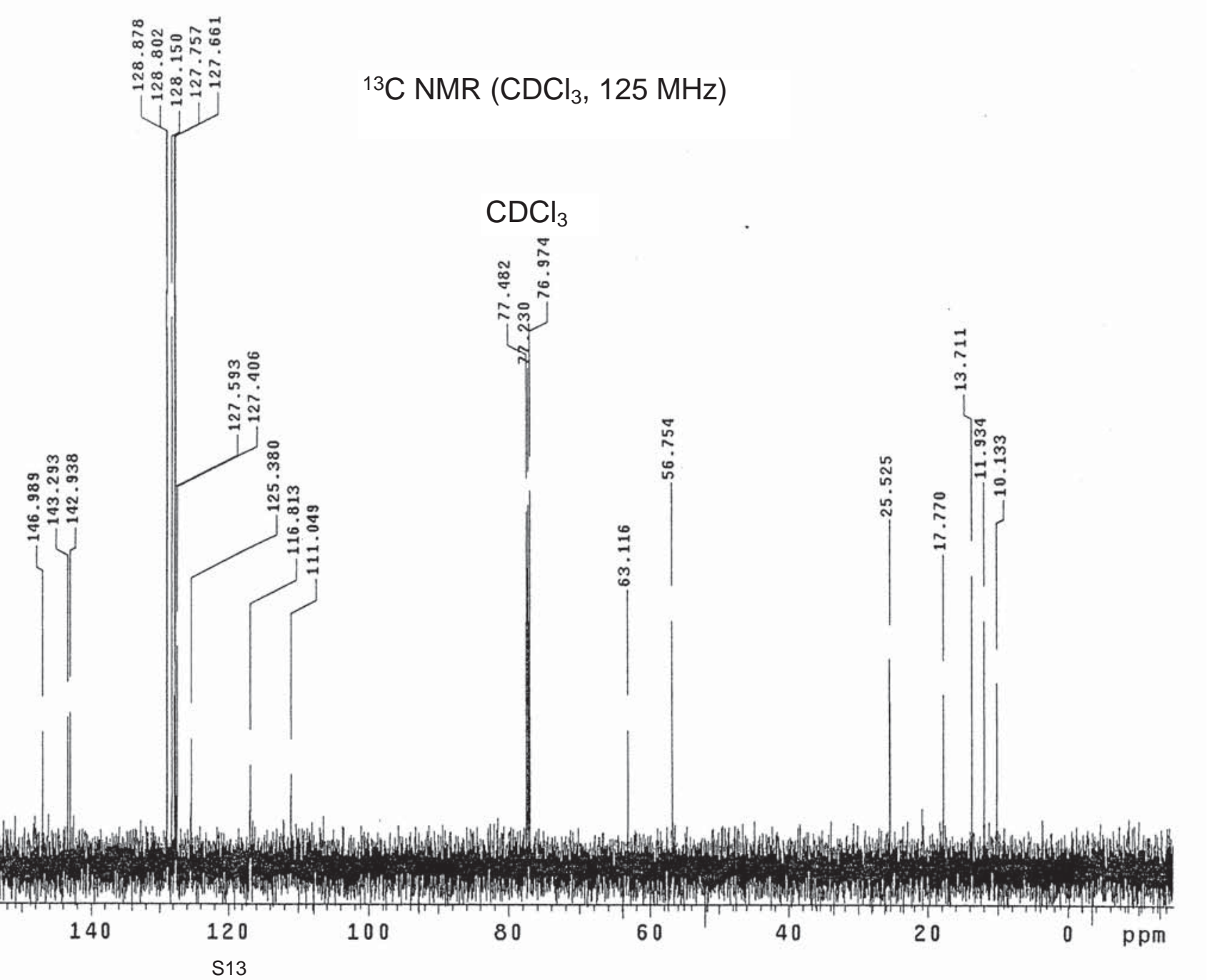

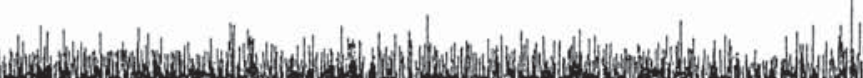




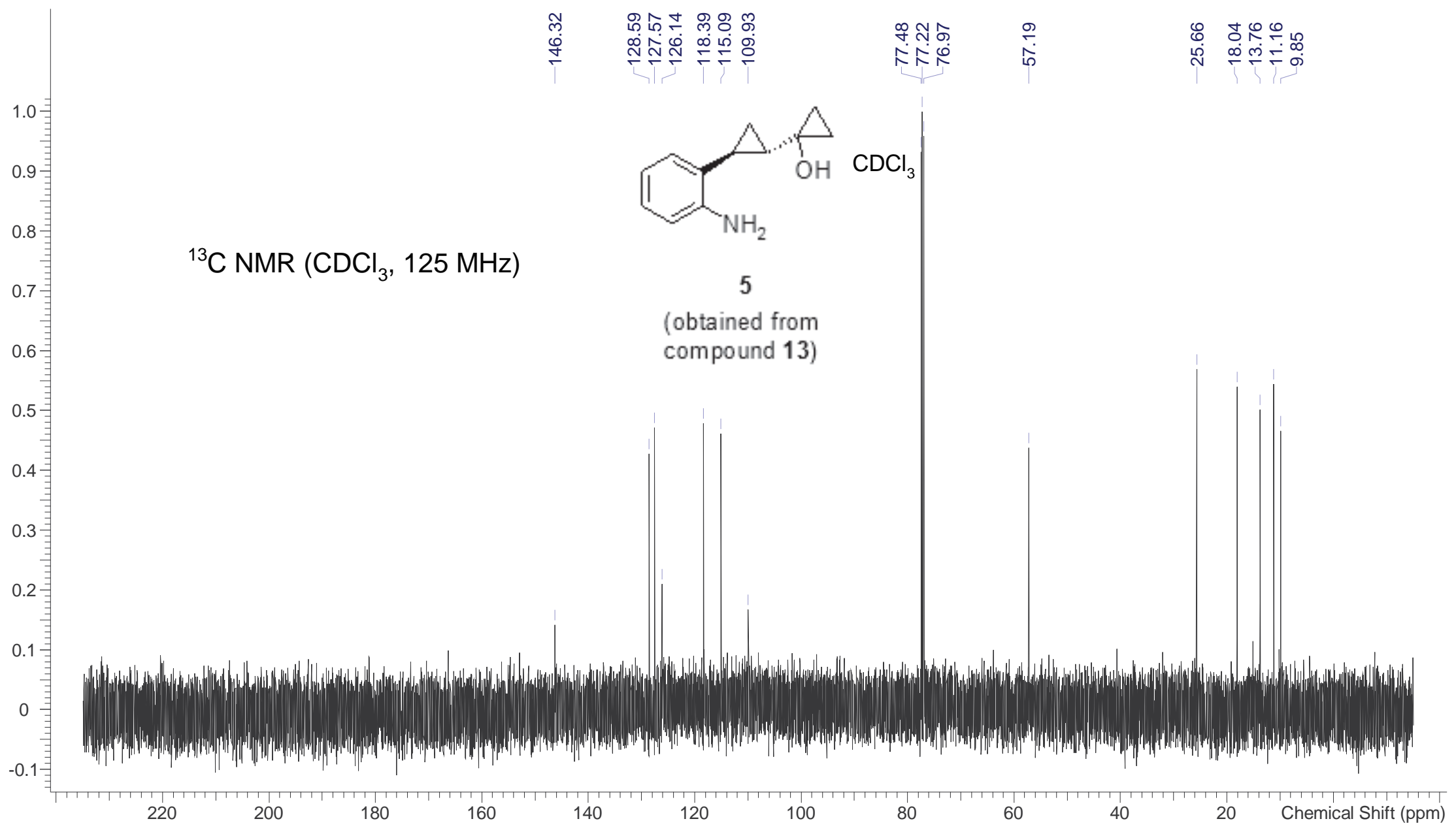




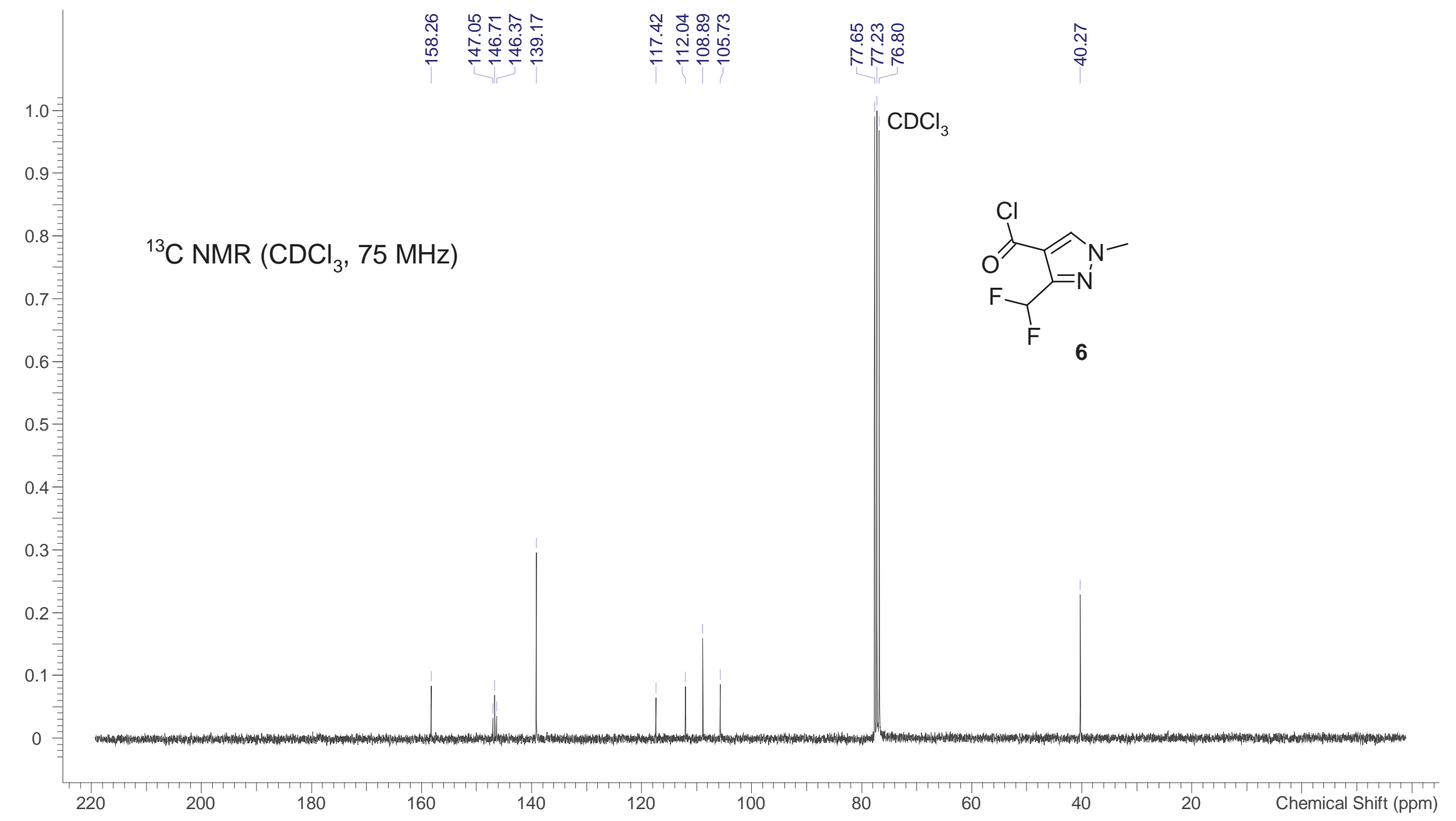




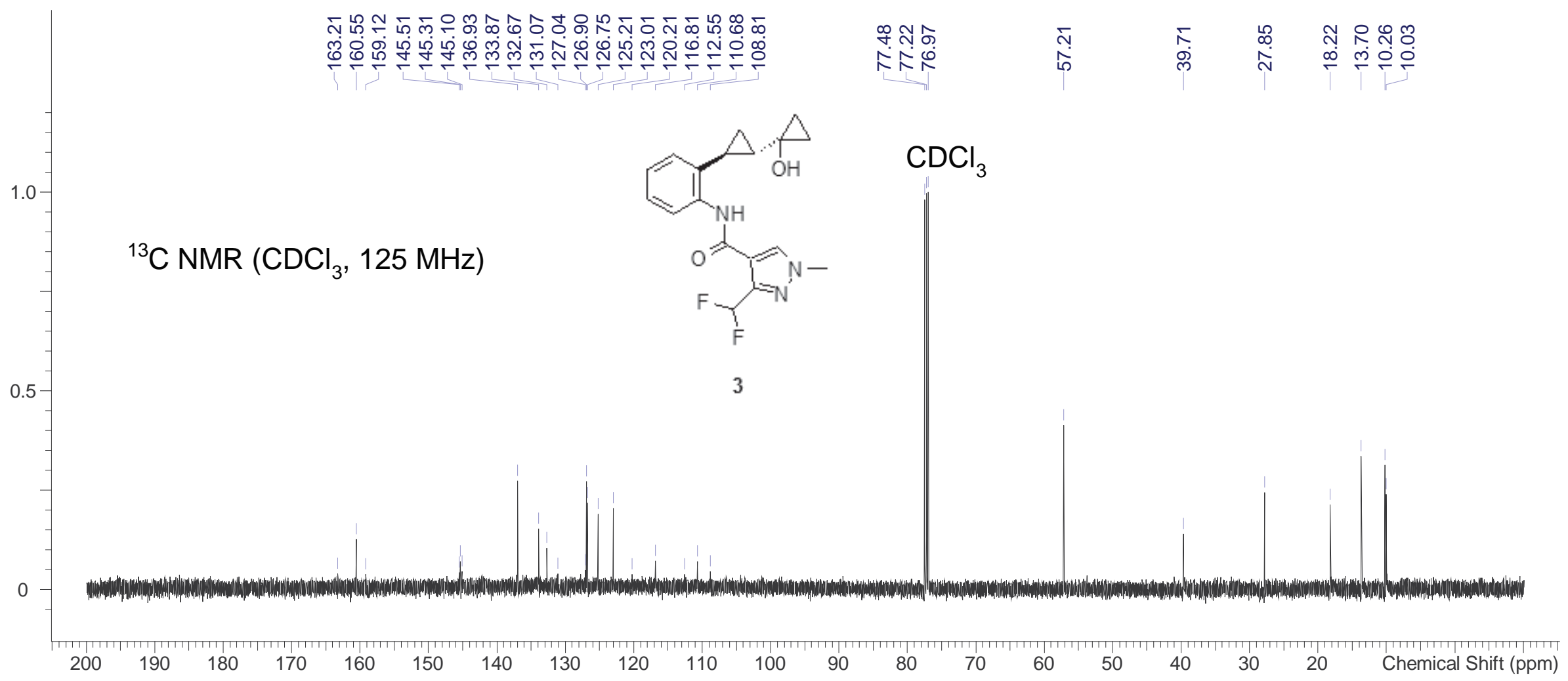




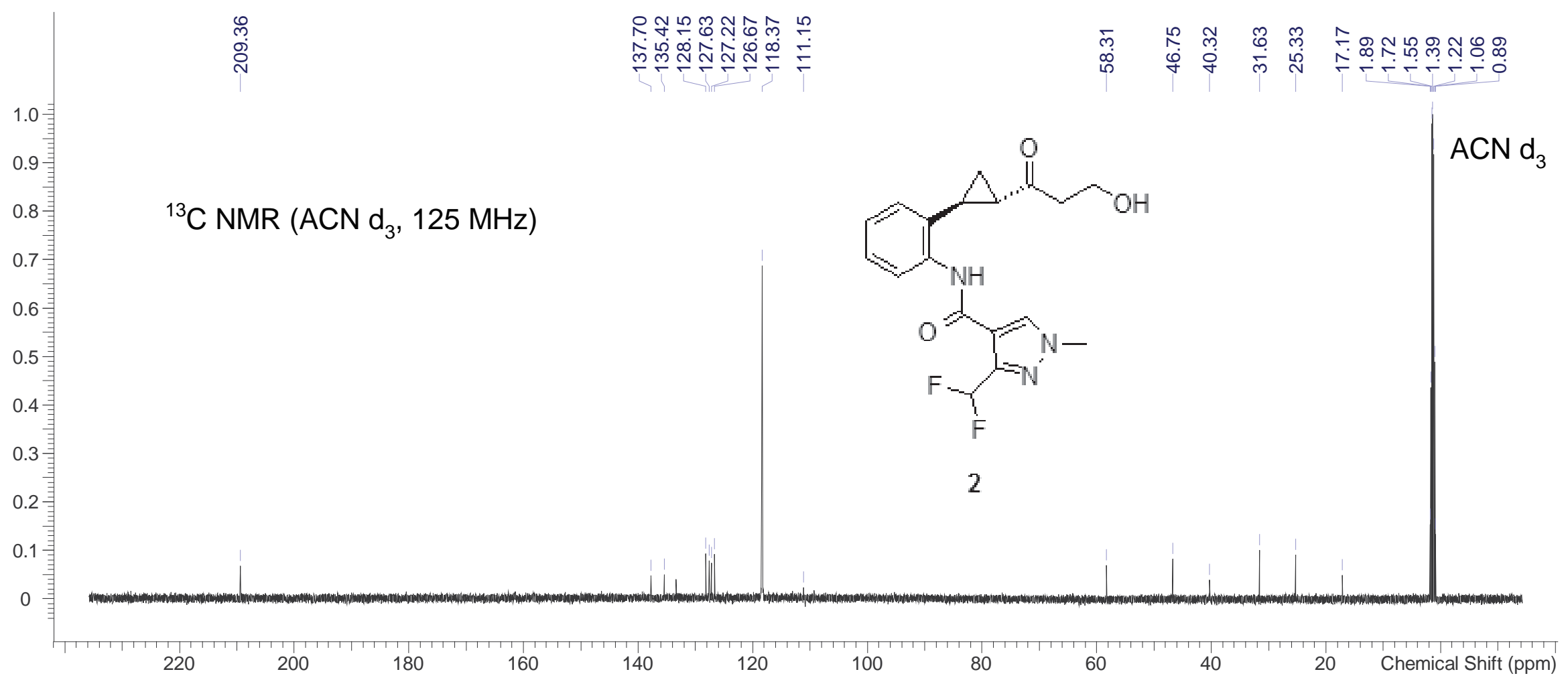

